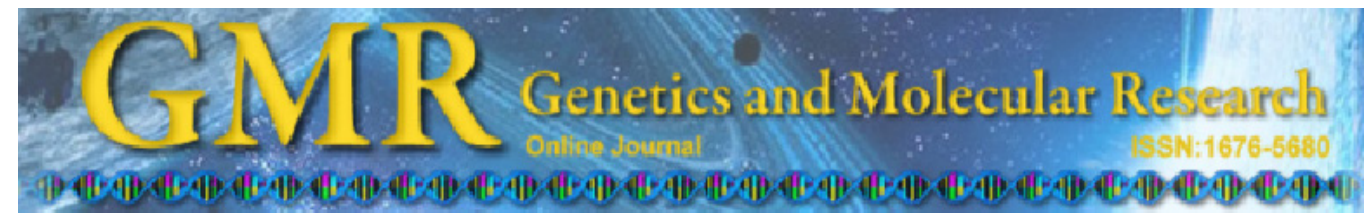

\title{
Isolation and characterization of the organ-specific and light-inducible promoter of the gene encoding rubisco activase in potato (Solanum tuberosum)
}

D. Qu*, Y. Song*, W.M. Li, X.W. Pei, Z.X. Wang, S.R. Jia and Y.Q. Zhang

Biotechnology Research Institute, National Key Facility for Gene Resources and Gene Improvement, Chinese Academy of Agricultural Sciences, Beijing, P.R. China

*These authors contributed equally to this study.

Corresponding author: Y.Q. Zhang

E-mail: zhangyongq@sina.com

Genet. Mol. Res. 10 (2): 621-631 (2011)

Received November 24, 2010

Accepted February 1, 2011

Published April 12, 2011

DOI 10.4238/vol10-2gmr1088

ABSTRACT. Constitutive promoters have been widely used in crop
biotechnology applications. Tissue-specific or inducible promoters,
however, have advantages in some cases. We isolated the 731-bp 5'
flanking sequence of a potato (Solanum tuberosum) gene, encoding
ribulose-1,5-bisphosphate carboxylase/oxygenase (rubisco) activase
( $R C A$ ), which was isolated by genome walking. By using GUS as
a reporter and with Northern blot analysis, the 702 -bp fragment
(referred to as $S t R C A \mathrm{p}$ ), ranging from nt -731 to -30 relative to the
initiation code of the $R C A$ gene, was analyzed in transgenic tobacco
plants. The activity of $S t R C A \mathrm{p}$ in leaves was 0.4 -fold less than that of
cauliflower mosaic virus $35 \mathrm{~S}$ promoter, and was expressed throughout
the green part of the light-grown transgenic $\mathrm{T}_{1}$ seedlings, including
cytoledons, leaves and young stems, but not roots. Further deletion
analysis revealed that a shorter fragment (nt -249 to -30, StRCAp2)
retained light-inducible features in cytoledons and leaves, but showed 
no detectable activity in young stems and roots. Although the activity of StRCAp2 in leaves was reduced significantly compared with that of $S t R C A \mathrm{p}$, the overall data indicated that cis-elements sufficient to regulate organ-specific and light-inducible transcription are within the 220-bp fragment. There is potential for application of StRCAp in plant genetic engineering.

Key words: Solanum tuberosum; Organ-specific and light-inducible; Promoter; Rubisco activase

\section{INTRODUCTION}

Rubisco activase (RCA) belongs to the AAA+ family of ATPases associated with diverse cellular activities (Portis Jr., 2003), and is of a chloroplast-localized enzyme that catalyzes the activation of ribulose-1,5-bisphosphate carboxylase/oxygenase (Rubisco). To date, a large number of $R C A$ genes have been isolated from over 10 species, including monocotyledon [barley (Rundle and Zielinski, 1991); rice (To et al., 1999); wheat (Law and Crafts-Brandner, 2001); maize (Ayala-Ochoa et al., 2004)], dicotyledon [spinach (Werneke et al., 1988); Arabidopsis thaliana (Pilgrim and McClung, 1993); apple (Watillon et al., 1993); tobacco (Qian and Rodermel, 1993); cotton (Salvucci et al., 2003); soybean (Yin et al., 2010); sweet potato (Xu et al., 2010)] as well as Chlamydomonas reinhardtii (Roesler and Ogren, 1990), and the functional model of RCA protein in photosynthesis has been well established especially in recent years (Portis Jr., 2003; Salvucci and CraftsBrandner, 2004; Salvucci et al., 2006; Salvucci, 2008; Portis Jr. et al., 2008).

In most plant species studied, RCA presents $\alpha$ - and $\beta$-isoforms. The two forms differ only at the C-terminus (Salvucci et al., 1987; Portis Jr., 2003; Portis Jr. et al., 2008) and arise from the same gene by alternative splicing (Werneke et al., 1988; Pilgrim and McClung, 1993; To et al., 1999), or are produced from related genes (Salvucci et al., 2003). Alternatively, some plants, such as maize, tobacco, bean, and cucumber, only express the $\beta$-isoform of RCA (Portis Jr., 2003). Nonetheless, the data collectively demonstrate that the expression of $R C A$ transcripts is only in the green part of higher plants and light regulated (Rundle and Zielinski, 1991; Orozco and Ogren, 1993; Watillon et al., 1993; Liu et al., 1996; Xu et al., 2010), indicating that the $R C A$ promoters are organ-specific as well as light-inducible.

In contrast to the $R C A$ gene and its encoding protein, which has been well studied in a few species, the $R C A$ promoters have so far only been isolated and functionally analyzed in spinach (Orozco and Ogren, 1993) and Arabidopsis (Liu et al., 1996). Consistent with the transcriptional pattern of the $R C A$ gene, both promoters are only active in the green part of plants and light-inducible. Deletion mapping showed that the cis-elements sufficient to confer organ-specific and light-regulated transcription in both $R C A$ promoters are localized proximal to the transcription start site (Orozco and Ogren, 1993; Liu et al., 1996).

So far, constitutive promoters, in particular the cauliflower mosaic virus (CaMV) $35 \mathrm{~S}$ promoter, are widely used in plant genetic engineering. It has been accepted, however, that constitutive expression of foreign proteins may impose extra metabolic burden as well as other adverse effects on transgenic plants, finally resulting in abnormal development 
(Shelton et al., 2002). In this regard, the organ-specific and light-regulated promoters, such as the $R C A$ promoter, have great potential for use in crop biotechnology applications.

Potato (Solanum tuberosum L.) is one of the most important crops in the world and ranks fourth after rice, wheat and maize in production. As a kind of heliophyte species, the growth of potato depends greatly on light. The potato $R C A$ gene, as well as its promoter sequence, however, remain to be determined. In this study, we isolated the 731-bp 5' flanking sequence of the $R C A$ open reading frame (ORF) from potato, and demonstrated that this fragment had promoter activity with organ-specific and lightinducible features. Furthermore, we determined that a 220-bp short sequence adjacent to the site of transcription initiation was sufficient to confer transcriptional response to both organ-specific cues and light.

\section{MATERIAL AND METHODS}

\section{Isolation of the $R C A$ promoter in potato}

Total RNA was firstly extracted from the leaves of a cultivated potato species, Atlantic (Chomczynski and Sacchi, 1987), then reverse transcription-polymerase chain reaction (RT-PCR) was performed to amplify the internal part of the $R C A$ gene with a pair of degenerate primers (5'-TTCCHCTYATYYTKGGTRTHTGGG-3' and 5'-TCACYTCRTCRTCRTANACHCKG GC-3'), resulting in a 603-bp PCR product. According to the nucleotide sequence of the partial $R C A$ gene, three corresponding primers were designed (5' phosphate-labeled primer: 5'-PCACTTCATCGTCA-3'; the first pair of primers: 5'-GTTCTACTGGGCACCAACTAGG-3' and 5'-GGTTGATTCCCATCTTTCTGAAG-3'; the nested pair of primers: 5'-GATTTCTTCGGT GCTTTGAGGG-3' and 5'-CATACACCCAAGATGAGTGGAA-3'), and 5' RACE was further carried out to identify the 5 ' end of the $R C A$ transcript by following the manufacturer instruction (5'-Full RACE Core Set, TaKaRa, Japan).

Genome walking was performed subsequently to isolate the putative promoter region of the $R C A$ gene in potato accordingly (LA PCR ${ }^{\mathrm{TM}}$ in vitro Cloning Kit, TaKaRa). The genomic DNA from potato Atlantic was digested with Sau3AI, and ligated with adaptors to result in the template used subsequently. A nested-PCR was then performed to amplify the 5' flanking sequence of the $R C A$ gene with two pairs of primers (the first pair of primers: 5'-GTACATATT GTCGTTAGAACGCGTAATACGACTCA-3' and 5'-CGTTAGAACGCGTAATACGACT CACTATAGGGAGA-3'; the nested pair of primers: 5'-GAAGTTCTTGGTGATGTGAA CAGCAAGTTTG-3' and 5'-GGAAAAGACTGTCAACCATACCCTTACCCCT-3') designed according to the identified internal part of the $R C A$ gene as well as $5^{\prime}$ end sequence of the $R C A$ transcript. After gel purification, the resulting PCR products were inserted into T-vectors and transformed into Escherichia coli DH5a (Gibco-BRL). The 10 positive clones were subjected to DNA sequencing. Finally, the resulting sequences including the 5' part of the $R C A$ gene and the putative promoter region were assembled into one contiguous sequence, which was deposited in Genbank accession No. HQ259068.

\section{Construction of plant expression vectors}

To avoid the restriction digestion site of HindIII at nt -26 (the adenine in initiation 
code ATG is assumed to be $n t+1$ ), the fragment ranging from nt -731 to -30 (referred to as $S t R C A \mathrm{p}$ hereafter) of the putative $R C A$ promoter region was subjected to PCR amplification by using a primer pair (5'-CGCAAGCTTAAATCTAACCTCATA-3' and 5'-ACGTCTAG ATTCTTGATTTGCCTTA-3', the HindIII and BamHI sites were underlined). The resulting PCR products were purified and double digested with HindIII and BamHI, then inserted into the HindIII/BamHI-digested pBI121 (Clontech) to generate pRCAp (Figure $1 \mathrm{~A})$, in which the transcription of the uidA gene encoding GUS reporter was driven by $S t R C A \mathrm{p}$, rather than the original CaMV $35 \mathrm{~S}$ promoter. With the same strategy, two truncated versions of $S t R C A \mathrm{p}$, which range from nt -498 to -30 , and $\mathrm{nt}-249$ to -30 , were introduced into pBI121 to result in pRCAp1 and pRCAp2 carrying the expression cassette of StRCAp1-uidA or StRCAp2-uidA, respectively (Figure 1A), for identification of the shorter $R C A$ promoter fragment sufficient to confer the organ-specific and lightinducible activity.

A

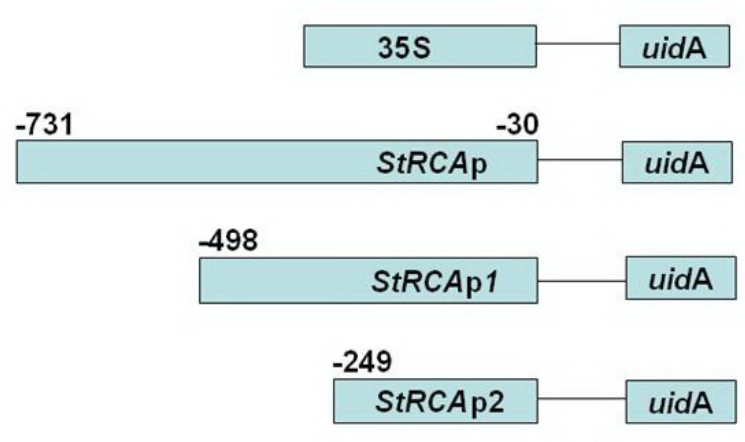

B

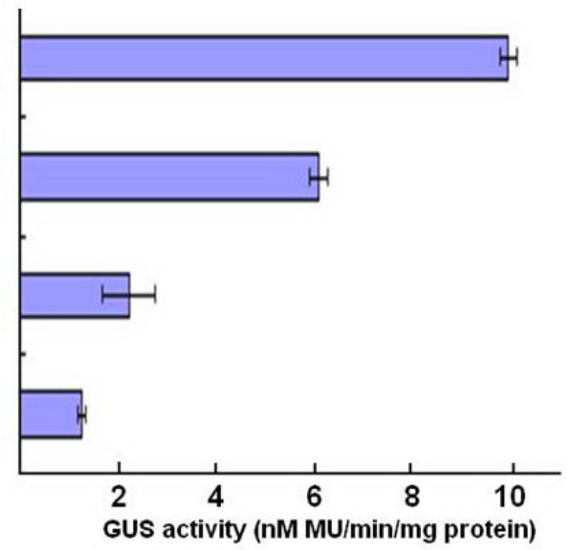

Figure 1. A. Schematic diagrams of the expression cassettes of StRCAp-uidA, StRCAp1-uidA and StRCAp2uidA, which were generated by fusion of the 5'-flanking regions (from nt -731 to $-30,-498$ to -30 , and -249 to -30 ) of the potato $R C A$ gene with the uidA gene. B. Horizontal bars represent the average GUS activities in fully expanded leaves, which were collected from different groups of transgenic $\mathrm{T}_{1}$ tobacco plants at 5- or 6-leaf stage. Each group included at least 10 individual plants. The average GUS activity driven by CaMV $35 \mathrm{~S}$ promoter was measured as a control.

\section{Generation of transgenic tobacco plants}

The three plant expression vectors as well as pBI121 were introduced into the Agrobacterium tumefaciens strain LBA4404 by freeze-thaw. The transgenic plants were generated by Agrobacterium-mediated transformation on leaf disks of Nicotiana tabacum cv. NC89 as described (Anonymous, 1985). The regenerated kanamycin-resistant plants $\left(\mathrm{T}_{0}\right)$ confirmed by PCR and GUS histochemical staining (Jefferson et al., 1987) were grown in a greenhouse, and allowed to generate seeds via self-pollination. 


\section{Light treatment, Northern blot analysis and GUS activity assay}

Self-pollinated seeds were planted on selection plates containing Murashige and Skoog salts (Murashige and Skoog, 1962), and $200 \mathrm{mg} / \mathrm{L}$ kanamycin at $25^{\circ} \mathrm{C}$. When the first or second leaf developed, the resistant seedlings were halved and treated with continuous white light and dark, respectively. After 7 days, 10 seedlings were randomly selected from each treatment and subjected to histochemical GUS assay as described by Jefferson et al. (1987). The other etiolated seedlings in the dark were exposed to a light intensity of $2000 \mathrm{~lx}$, and the second leaves were collected from individual seedlings at 0 -, 8-, 16-, and 24-h intervals, respectively, to extract total RNA for Northern blot analysis with the labeled uidA-specific probe (Xie et al., 2003); while the other light-grown seedlings were transferred to soil and cultured in a green house, the fully expanded leaves were sampled at 5- or 6-leaf stage for quantitative GUS assay (Jefferson et al., 1987).

\section{Computer analysis of the isolated $R C A$ promoter sequence}

The nucleotide sequence of the isolated $R C A$ promoter was aligned by using the BLAST algorithm (Altschul et al., 1997). The putative cis-acting-regulatory elements within that sequence were searched against both PlantCARE and PLACE Databases (Prestridge, 1991; Higo et al., 1999; Lescot et al., 2002).

\section{RESULTS}

\section{Isolation and sequence analysis of the $R C A$ promoter in potato}

To isolate the $R C A$ promoter in potato, we firstly cloned the $5^{\prime}$ end of the $R C A$ transcript with the length of 1146 bp (Genbank accession No. HQ259068). The experiment of 5' RACE determined that one adenine at nt -68 relative to the initiation code was the transcription start site, indicating that the $5^{\prime}$ untranslation region of $R C A$ was $68 \mathrm{bp}$ in length (Figure 2). Based on the identified partial sequence of the $R C A$, a 731-bp 5' flanking sequence of the potato $R C A$ gene was isolated by using genomic walking (Figure 2; Genbank accession No. HQ259068). When using BLAST to search the nucleotide database in Genbank, several sequences (for instance, AC151803.1 and AC231666.1) from plants of genus Solanum were found to share some similarity with the 731-bp sequence, but without function annotation. A 'TATA' box motif 'TTATTT' at nt -62 and a 'CAAT' box at nt -117 were predicted by searching against the databases of PlantCARE and PLACE (Figure 2). Meanwhile, numbers of conserved light-responsive motifs, such as 'I' box (Terzaghi and Cashmore, 1995) and 'GATA' box (Gilmartin et al., 1990), were identified within the 731bp fragment (data not shown), suggesting the possible role of this fragment.

\section{Transcriptional activity of $R C A$ promoter is organ-specific and light-inducible}

To verify the transcriptional activity of the isolated fragment, the transgenic tobacco NC89 plants were transformed with A. tumefaciens, carrying pRCAp. Histochemical staining revealed the strong GUS activity in transgenic $\mathrm{T}_{0}$ tobacco leaves (data not shown), providing 


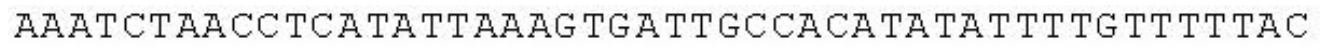

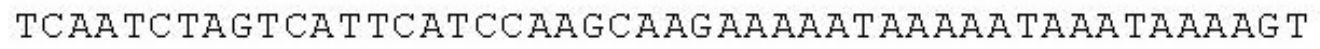

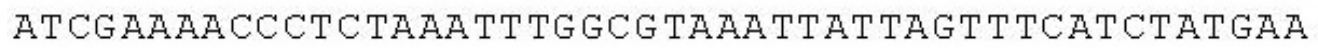
GTATTGACAG TCTTAACAATACCTCTCTACTTGACTAACTAAACTTAG ATACACCCСTGATCTGCCACATGACATAGCAAGTGGTCTCAAACTCT T GTAGGAGCATGAAACTTTTAATAAAAAGACGAGAGGAGTGTTGAAAAT

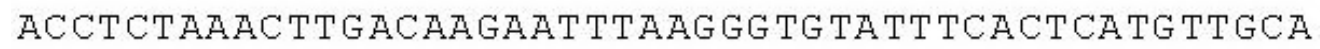
AGGTCCGGGGTATATTAAGTTCAGTTAGTCAAATAAAGTGGTATTTTT

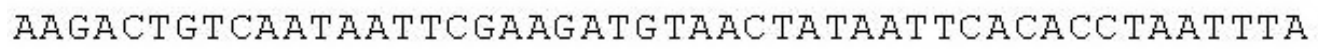

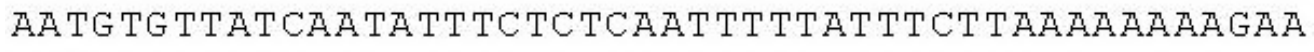

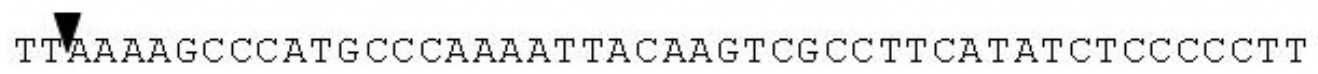
AG ATAACСАATCT TAATT TG TGGCCATTGAGTAAAG TTAAGCCAAAT

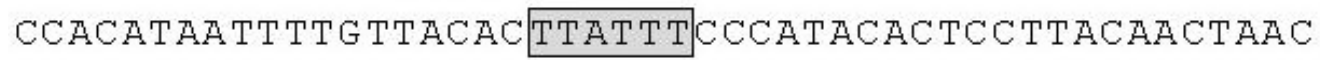

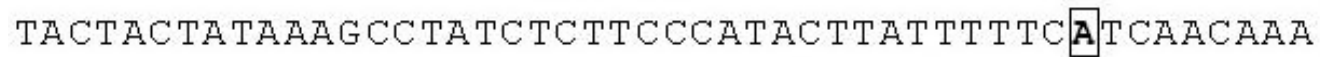
TTTTTGAдATTTCTAдGGCAAATCAAGAдAGCTTTTTTCATCAдTTTT AGTCATCAAGAATG GCT GCC ACA GTG TCA ACC ATT GGA

\section{$\begin{array}{llllllllll}\text { M } & \text { A } & \text { A } & \text { T } & \text { V } & \text { S } & \text { T } & \text { I } & \text { G }\end{array}$}

Figure 2. The nucleotide sequence of the 5'-flanking regions (from nt -731 to +27 ) of the potato $R C A$ gene determined in this study. Putative cis-acting elements including 'TATA' box and 'CAAT' box were masked with gray. The putative translation start site, ATG, was indicated in bold, and one boxed adenine at nt -68 was identified as the transcription initiation site. The black filled triangles indicate the positions of $\mathrm{nt}$ $-498,-249$ and -30 , respectively. The unique restriction site of HindIII within this sequence is underlined.

evidence that $S t R C A \mathrm{p}$, the fragment ranging from nt -731 to -30 , was able to drive the expression of uidA gene in tobacco.

To better evaluate the promoter activity of StRCAp, self-pollinated seeds from the transgenic $\mathrm{T}_{0}$ plants were germinated, and the resulting kanamycin-resistant $\mathrm{T}_{1}$ seedlings were used in the following experiments. Since the $R C A$ promoters in both Arabidopsis and spinach are organ-specific and light-inducible (Orozco and Ogren, 1993; Liu et al, 1996), the transcriptional pattern of the StRCAp was investigated by using the light- and darkgrown seedlings. Histochemical staining showed that, in contrast to the constitutive GUS expression driven by CaMV 35S promoter (Figure 3A), StRCAp failed to drive the GUS expression in dark-grown transgenic seedlings (data not shown but as in Figure 3B), whereas it was active throughout the whole green part of light-grown ones including cotyledons, leaves and young stems, but not roots (Figure 3C). As expected, 


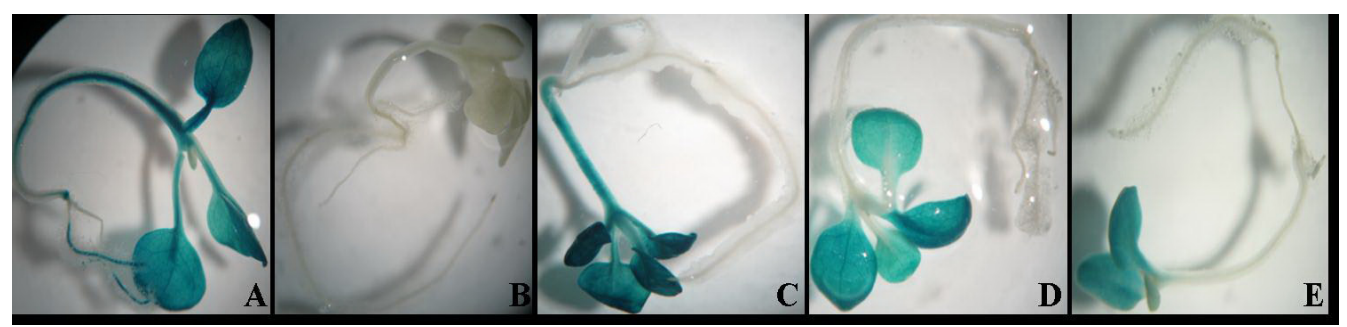

Figure 3. Histochemical localization of GUS expression in transgenic $\mathrm{T}_{1}$ tobacco seedlings carrying the expression cassette of $R C A$-uidA. A. CaMV $35 \mathrm{~S}$ promoter drives GUS expression throughout the whole seedling. B. GUS activity was absent in wild-type tobacco seedlings whether under continuous light or in dark for 7 days. Similar results have been observed from the seedlings carrying StRCApuidA, StRCAp1-uidA or StRCAp2-uidA, which showed no GUS expression when grown in dark (data not shown). C. D. and E. The light-grown transgenic seedlings carrying StRCAp-uidA, StRCAp1uidA and StRCAp2-uidA, respectively, showed significant GUS activity. Notably, the StRCAp drove uidA expression in cytoledons, leaves and stems, whereas both StRCAp1 and StRCAp2 directed uidA expression in cytoledons and leaves, but not stems.

no GUS activity was detected in either light- or dark-grown tobacco NC89 seedlings (Figure 3B).

The subsequent Northern blot analysis confirmed the light-inducible feature of StRCAp. Consistent with the results of histochemical staining, no detectable uidA transcripts were accumulated in dark-grown seedlings carrying the StRCAp-uidA fusion (Figure 4A, lane 1). However, when the dark-grown seedlings were transferred to a light intensity of $2000 \mathrm{~lx}$, the accumulation of uidA transcripts could be detected at a 8-h exposure, and increased progressively following the increasing exposure times (Figure 4A, lanes 2-4).

Considering the organ-specific transcriptional feature of $S t R C A \mathrm{p}$, quantitative GUS activity analysis was performed only with the fully expanded leaves (without the main vein) of the transgenic $\mathrm{T}_{1}$ plants, which were cultured under continuous white light to 5- or 6-leaf stage. The results showed that the mean GUS activity driven by the $S t R C A \mathrm{p}$ was 0.4 -fold less than that of the CaMV $35 \mathrm{~S}$ promoter (Figure $1 \mathrm{~B}$ ).

\section{A 220-bp fragment of $R C A$ promoter sufficient for organ-specific and light-inducible expression}

The cis-element(s) conferring organ-specific and light-inducible activity was mapped by progressive deletion from the 5' end of StRCAp. To retain the putative 'CAAT' box (nt -185) and 'TATA' box (nt -130), two constructs harboring the smaller StRCAp fragments ranging from nt -498 to -31 (StRCAp1), and nt -289 to -31 (StRCAp2), respectively, were produced and used to generate the transgenic tobacco lines carrying StRCAp1-uidA and StRCAp2-uidA, respectively. Histochemical staining analysis revealed that no GUS activity was detected in either kind of dark-grown transgenic $\mathrm{T}_{1}$ seedling (data not shown but as in Figure 3B), whereas GUS activity was detected in light-grown ones of cotyledons and leaves, instead of young stems and roots (Figure 3D and E). 


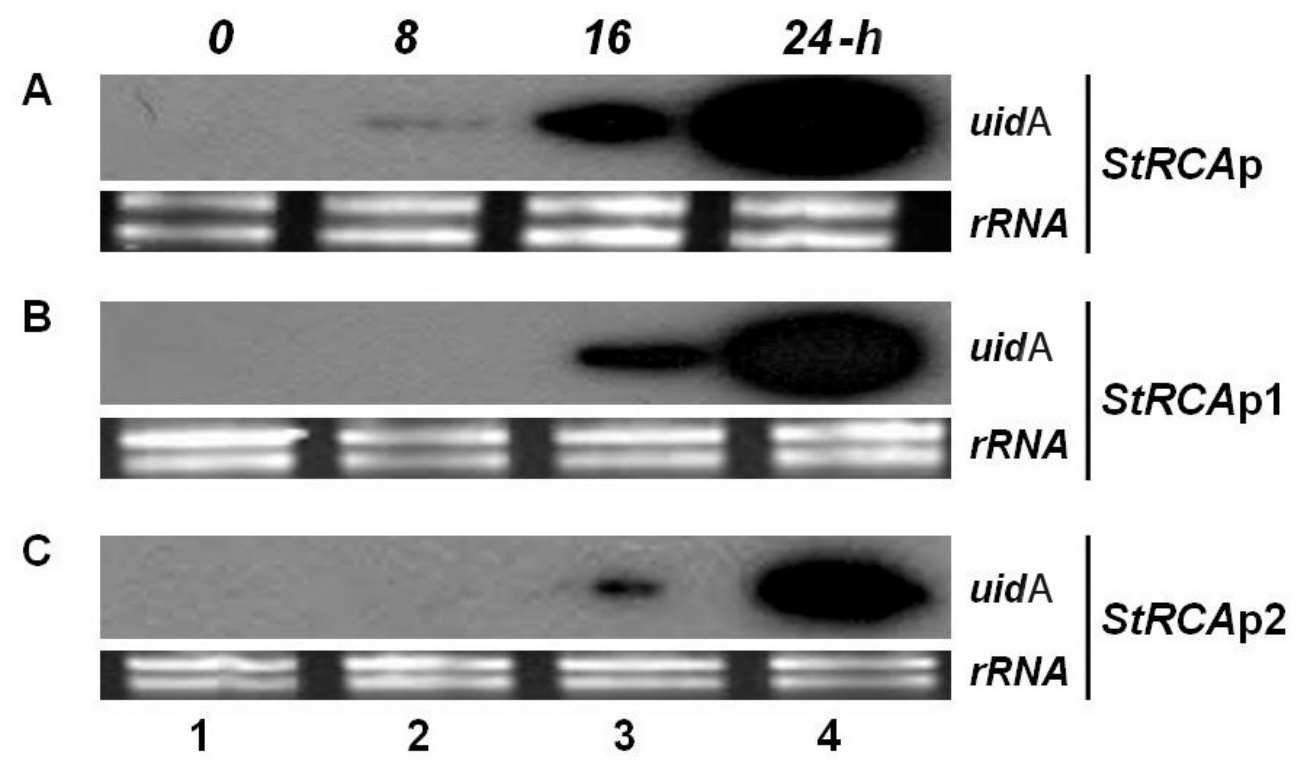

Figure 4. Accumulation of uidA mRNA in transgenic $\mathrm{T}_{1}$ tobacco seedlings carrying A. StRCAp-uidA, B. StRCAp1uidA or C. StRCAp2-uidA. The seedlings were first etiolated in the dark for 7 days, then exposed to a light intensity of 2000 lx for $0-, 8-, 16-$, and 24-h intervals, respectively. Total RNA extracted from the second leaves was loaded for Northern blot analysis by using a uidA-specific probe. Bands corresponding to the transcripts of uidA are indicated. The rRNAs are shown to indicate the relative equivalency of the samples loaded.

The light-inducible expression of both StRCAp1 and StRCAp2 was further verified by Northern blot analysis. The uidA transcripts were under the detectable level in dark-grown seedlings carrying either StRCAp1-uidA or StRCAp2-uidA (Figure 4B and C, lane 1). However, when the seedlings were transferred to a light intensity of $2000 \mathrm{~lx}$, the expression of uidA transcripts was increased stepwise following the prolonged exposure times (Figure 4B and C, lanes 2-4), like the behavior of StRCAp under the light treatment (Figure 4A). It was noteworthy, however, that the accumulation of uidA was not detected until after $16 \mathrm{~h}$ of continuous exposure when driven by either StRCAp1 or StRCAp2, suggesting that removal of the 5' end of $S t R C A \mathrm{p}$ greatly affected its transcriptional activity.

The reduced activities of StRCAp1 and StRCAp2 in leaves were confirmed by quantitative GUS activity analysis. The results showed the two truncated fragments retained the promoter activities, which, however, were only $\sim 37$ and $\sim 23 \%$ compared with that of StRCAp. Nonetheless, the collective data established that the fragment extracted from $\mathrm{nt}-249$ to -30 (StRCAp2) was sufficient to drive GUS expression in light-inducible and organ-specific transcriptional manner.

\section{DISCUSSION}

In this study, a 731-bp fragment upstream of the initiation code of the $R C A$ gene in potato was isolated and analyzed. GUS activity assay and Northern blot analysis performed with transgenic tobacco plants revealed that StRCAp, ranging from nt -731 to -30 , was able 
to drive the uidA gene expression in light but not darkness. Additionally, the GUS activity was not detectable in roots, but throughout the cytoledons, leaves and young stems, the whole green part of tobacco seedling. Taken together, these data clearly support the StRCAp from potato as a promoter with light-inducible and organ-specific features, resembling the behavior of $R C A$ promoters in Arabidopsis and spinach reported previously (Orozco and Ogren, 1993; Liu et al., 1996).

It has been established that, for the $R C A$ promoter from either Arabidopsis or spinach, a $\sim 300$-bp fragment adjacent to the transcription start site is sufficient to confer light-inducible and organ-specific transcription (Orozco and Ogren, 1993; Liu et al., 1996). A similar result was obtained from the potato $R C A$ promoter, the cis-elements, which are necessary for lightinducible and organ-specific transcription also localized within a 220-bp fragment just upstream of the transcription start site, as evidence that two 5' progressively truncated versions, $S t R C A \mathrm{p} 1$ and StRCAp2, retained light-inducible and organ-specific manner in transgenic tobacco plant.

GUS activity analysis showed that the transcriptional activities of two truncated versions, StRCAp1 and StRCAp2, in leaves were reduced significantly compared with that of StRCAp, suggesting that the deleted 483-bp fragment might harbor cis-regulatory element(s) such as transcriptional enhancer(s) (Halfon, 2006). Except for the reduced promoter activity, both StRCAp1 and StRCAp2 were only active in cytoledons and leaves, unlike the full length of $S t R C A \mathrm{p}$, which drove the uidA expression in not only cytoledons and leaves, but also young stems. It has been determined that the $R C A$ transcripts in stem are much less abundant than those in leaves (Liu et al., 1996), that is, the activity of a mutated $R C A$ promoter might be completely shut down in stems but only reduced in leaves. In this case, therefore, it is conceivable that the failure of StRCAp1 and StRCAp2 to drive GUS expression in young stems might be correlated with their significantly reduced activity in leaves. However, we cannot rule out the possibility that the removed fragment from -731 to -249 might contain some yet-unknown cis-element(s) essential for regulation of StRCAp activity in young stems.

The light-inducible and organ-specific features of $S t R C A \mathrm{p}$ present specific advantages in plant genetic engineering for leaf-consuming pests or foliar disease resistance. For instance, in potato genetic engineering, StRCAp could be employed to express defensive genes only in the green part of plant to specifically resist Colorado beetle (Weber, 2003), powdery mildew (Park and Jones, 2008) or aphid, which transmit several important potato viruses (Hodgson, 1991). The application of StRCAp would not only reduce the metabolic burden imposed on transgenic potato plants, but also reduce concern over food safety (Stewart et al., 2000; Shelton et al., 2002) since the edible part of potato is a tuber that grows underground. Additionally, considering cisgenesis, a scientific theory proposed recently with regard to the genetically modified plants (Jacobsen and Schouten, 2007; Schouten and Jacobsen, 2008), extra values would be expected when the $S t R C A \mathrm{p}$ from a potato species was applied back to potato genetic engineering.

In summary, the isolated 702-bp fragment (StRCAp) upstream of the RCA ORF in potato showed inducible and organ-specific transcriptional activity, which, however, could be conferred by only the 220-bp short fragment proximal to the 3' end of StRCAp. So far, several light-inducible and organ-specific promoters have been determined (Ueda et al., 1989; Orozco and Ogren, 1993; Liu et al., 1996; Nomura et al., 2000; de Souza et al., 2009), while the characterization of StRCAp has provided an alternative promoter with similar characteristics for plant 
genetic engineering and shed some light on the yet-unknown $R C A$ transcriptional patterns in potato. Given the lower activity of StRCAp compared with that of CaMV 35S promoter, the ongoing study is focused on improving the activity of this promoter but without disturbing its light-inducible and organ-specific manner.

\section{ACKNOWLEDGMENTS}

Research was supported by grants from the National High-Tech Research and Development (863) Project (\#2002AA212061 and \#2008AA10Z158), and Special Fund for AgroScientific Research in the Public Interest (\#200803024).

\section{REFERENCES}

Altschul SF, Madden TL, Schaffer AA, Zhang J, et al. (1997). Gapped BLAST and PSI-BLAST: a new generation of protein database search programs. Nucleic Acids Res. 25: 3389-3402.

Anonymous (1985). A simple and general method for transferring genes into plants. Science 227: 1229-1231.

Ayala-Ochoa A, Vargas-Suarez M, Loza-Tavera H, Leon P, et al. (2004). In maize, two distinct ribulose 1,5-bisphosphate carboxylase/oxygenase activase transcripts have different day/night patterns of expression. Biochimie 86: 439-449.

Chomczynski P and Sacchi N (1987). Single-step method of RNA isolation by acid guanidinium thiocyanate-phenolchloroform extraction. Anal. Biochem. 162: 156-159.

de Souza CR, Aragao FJ, Moreira EC, Costa CN, et al. (2009). Isolation and characterization of the promoter sequence of a cassava gene coding for Pt2L4, a glutamic acid-rich protein differentially expressed in storage roots. Genet. Mol. Res. 8: 334-344.

Gilmartin PM, Sarokin L, Memelink J and Chua NH (1990). Molecular light switches for plant genes. Plant Cell 2: 369 378.

Halfon MS (2006). (Re)modeling the transcriptional enhancer. Nat. Genet. 38: 1102-1103.

Higo K, Ugawa Y, Iwamoto M and Korenaga T (1999). Plant cis-acting regulatory DNA elements (PLACE) database: 1999. Nucleic Acids Res. 27: 297-300.

Hodgson C (1991). Dispersal of apterous aphids (Homoptera: Aphididae) from their host plant and its significance. Bull. Entomol. Res. 81: 417-427.

Jacobsen E and Schouten HJ (2007). Cisgenesis strongly improves introgression breeding and induced translocation breeding of plants. Trends Biotechnol. 25: 219-223.

Jefferson RA, Kavanagh TA and Bevan MW (1987). GUS fusions: beta-glucuronidase as a sensitive and versatile gene fusion marker in higher plants. EMBO J. 6: 3901-3907.

Law RD and Crafts-Brandner SJ (2001). High temperature stress increases the expression of wheat leaf ribulose-1, 5-bisphosphate carboxylase/oxygenase activase protein. Arch. Biochem. Biophys. 386: 261-267.

Lescot M, Dehais P, Thijs G, Marchal K, et al. (2002). PlantCARE, a database of plant cis-acting regulatory elements and a portal to tools for in silico analysis of promoter sequences. Nucleic Acids Res. 30: 325-327.

Liu Z, Taub CC and McClung CR (1996). Identification of an Arabidopsis thaliana ribulose-1,5-bisphosphate carboxylase/ oxygenase activase (RCA) minimal promoter regulated by light and the circadian clock. Plant Physiol. 112: 43-51.

Murashige T and Skoog F (1962). A revised medium for rapid growth and bioassays with tobacco tissue cultures. Physiol. Plant. 15: 473-497.

Nomura M, Katayama K, Nishimura A, Ishida Y, et al. (2000). The promoter of rbcS in a C3 plant (rice) directs organspecific, light-dependent expression in a C4 plant (maize), but does not confer bundle sheath cell-specific expression. Plant Mol. Biol. 44: 99-106.

Orozco BM and Ogren WL (1993). Localization of light-inducible and tissue-specific regions of the spinach ribulose bisphosphate carboxylase/oxygenase (rubisco) activase promoter in transgenic tobacco plants. Plant Mol. Biol. 23: 1129-1138.

Park TH and Jones JDG (2008). A new resistance gene to powdery mildew identified in Solanum neorossii has been localized on the short arm of potato chromosome 6. Euphytica 166: 331-339.

Pilgrim ML and McClung CR (1993). Differential involvement of the circadian clock in the expression of genes required for ribulose-1,5-bisphosphate carboxylase/oxygenase synthesis, assembly, and activation in Arabidopsis thaliana. 
Plant Physiol. 103: 553-564.

Portis AR Jr (2003). Rubisco activase - rubisco's catalytic chaperone. Photosynth. Res. 75: 11-27.

Portis AR Jr, Li C, Wang D and Salvucci ME (2008). Regulation of rubisco activase and its interaction with rubisco. $J$. Exp. Bot. 59: 1597-1604.

Prestridge DS (1991). SIGNAL SCAN: a computer program that scans DNA sequences for eukaryotic transcriptional elements. Comput. Appl. Biosci. 7: 203-206.

Qian J and Rodermel SR (1993). Ribulose-1,5-bisphosphate carboxylase/oxygenase activase cDNAs from Nicotiana tabacum. Plant Physiol. 102: 683-684.

Roesler KR and Ogren WL (1990). Primary structure of Chlamydomonas reinhardtii ribulose 1,5-bisphosphate carboxylase/oxygenase activase and evidence for a single polypeptide. Plant Physiol. 94: 1837-1841.

Rundle SJ and Zielinski RE (1991). Organization and expression of two tandemly oriented genes encoding ribulose bisphosphate carboxylase/oxygenase activase in barley. J. Biol. Chem. 266: 4677-4685.

Schouten HJ and Jacobsen E (2008). Cisgenesis and intragenesis, sisters in innovative plant breeding. Trends Plant Sci. 13: $260-261$.

Salvucci ME (2008). Association of rubisco activase with chaperonin-60beta: a possible mechanism for protecting photosynthesis during heat stress. J. Exp. Bot. 59: 1923-1933.

Salvucci ME and Crafts-Brandner SJ (2004). Relationship between the heat tolerance of photosynthesis and the thermal stability of rubisco activase in plants from contrasting thermal environments. Plant Physiol. 134: 1460-1470.

Salvucci ME, Werneke JM, Ogren WL and Portis AR (1987). Purification and species distribution of rubisco activase. Plant Physiol. 84: 930-936.

Salvucci ME, van de Loo FJ and Stecher D (2003). Two isoforms of rubisco activase in cotton, the products of separate genes not alternative splicing. Planta 216: 736-744.

Salvucci ME, DeRidder BP and Portis AR Jr (2006). Effect of activase level and isoform on the thermotolerance of photosynthesis in Arabidopsis. J. Exp. Bot. 57: 3793-3799.

Shelton AM, Zhao JZ and Roush RT (2002). Economic, ecological, food safety, and social consequences of the deployment of bt transgenic plants. Annu. Rev. Entomol. 47: 845-881.

Stewart CN Jr, Richards HA and Halfhill MD (2000). Transgenic plants and biosafety: science, misconceptions and public perceptions. Biotechniques 29: 832-843.

Terzaghi WB and Cashmore AR (1995). Light-regulated transcription. Annu. Rev. Plant Physiol. Plant Mol. Biol. 46: 445-474.

To KY, Suen DF and Chen SC (1999). Molecular characterization of ribulose-1,5-bisphosphate carboxylase/oxygenase activase in rice leaves. Planta 209: 66-76.

Ueda T, Pichersky E, Malik VS and Cashmore AR (1989). Level of expression of the tomato rbcS-3A gene is modulated by a far upstream promoter element in a developmentally regulated manner. Plant Cell. 1: 217-227.

Watillon B, Kettmann R, Boxus P and Burny A (1993). Developmental and circadian pattern of rubisco activase mRNA accumulation in apple plants. Plant Mol. Biol. 23: 501-509.

Weber D (2003). Colorado bettle: pest on the move. Pestic. Outlook 14: 256-259.

Werneke JM, Zielinski RE and Ogren WL (1988). Structure and expression of spinach leaf cDNA encoding ribulose bisphosphate carboxylase/oxygenase activase. Proc. Natl. Acad. Sci. U. S. A. 85: 787-791.

Xie Y, Liu Y, Meng M, Chen L, et al. (2003). Isolation and identification of a super strong plant promoter from cotton leaf curl Multan virus. Plant Mol. Biol. 53: 1-14.

$\mathrm{Xu} \mathrm{K}, \mathrm{He} \mathrm{B}, \mathrm{Zhou} \mathrm{S,} \mathrm{Li} \mathrm{Y,} \mathrm{et} \mathrm{al.} \mathrm{(2010).} \mathrm{Cloning} \mathrm{and} \mathrm{characterization} \mathrm{of} \mathrm{the} \mathrm{rubisco} \mathrm{activase} \mathrm{gene} \mathrm{from} \mathrm{Ipomoea} \mathrm{batatas}$ (L.) Lam. Mol. Biol. Rep. 37: 661-668.

Yin Z, Meng F, Song H, Wang X, et al. (2010). Expression quantitative trait loci analysis of two genes encoding rubisco activase in soybean. Plant Physiol. 152: 1625-1637. 